\title{
OA03 I-05. HIV escape from natural killer cytotoxicity: Nef inhibits NKp44L expression on HIV-infected CD4+ T cells H Fausther-Bovendo ${ }^{2}$, N Sol-Foulon ${ }^{1}$, O Schwartz ${ }^{1}$, P Debre ${ }^{2}$ and V Vieillard $* 2$
}

Address: ${ }^{1}$ Département de Virologie; Institut Pasteur, Paris, France and ${ }^{2}$ INSERM UMR945, Paris, France

* Corresponding author

from AIDS Vaccine 2009

Paris, France. 19-22 October 2009

Published: 22 October 2009

Retrovirology 2009, 6(Suppl 3):O23 doi:10.1/86/1742-4690-6-S3-O23

This abstract is available from: http://www.retrovirology.com/content/6/S3/O23

(c) 2009 Fausther-Bovendo et al; licensee BioMed Central Ltd.

\section{Background}

HIV infection leads to a progressive depletion of uninfected CD4 cells. We showed that NKp44L, a cellular ligand for an activating NK receptor is over-expressed during HIV infection and is correlated with both CD4 depletion and increase in viral load. NKp44L+CD4+ cells are highly sensitive to the NK lysis activity. However, HIV-infected cells are resistant to NK killing, suggesting that HIV-1 develop escape mechanisms to counteract NK cytotoxicity.

\section{Methods}

The role of Nef was determined using recombinant vaccines expressing different HIV-1 proteins and then a panel of isogenic viruses that expresses an array of established Nef mutants.

Functional NK activities were determined by $51 \mathrm{Cr}$ release assay, and degranulation capacities.

\section{Results}

During HIV infection, we show that NKp44L expression is restricted to uninfected cells. We demonstrate that Nef protein inhibits NKp44L expression on HIV-infected cells and their subsequent sensitivity to NK lysis. These data were confirmed with HIV-1 strains deleted for Nef, which induced an increased expression of NKp44L associated with high NK lysis sensitivity. Furthermore, our results provide evidence that Nef is required to block the translocation of NKp44L to the CD4 cell surface level.

\section{Conclusion}

We show here that Nef protein induced the dysregulation of NKp44L surface expression on CD4 cells. This novel escape mechanism could then play a key role in maintaining the HIV reservoir to ovoid recognition by NK cells. Thus, HIV induces in parallel the destruction of non-infectious cells, which over-expressed NKp44L, and the capacity for a long-term viral persistence, by the down modulation of NKp44L cell-surface translocation through Nef expression. Therapeutic intervention against Nef could be a novel strategy to destroy HIV-1 reservoir by a targeted attack mediated by NK cells. 